\title{
Synthesis of Four-Link Basic Kinematic Chains [BKC] with Spherical Pairs for Spatial Mechanisms
}

\author{
Algazy Zhauyt \\ Serikbay Kosbolov \\ Beibit Shingissov \\ Assylkhan Alymbetov \\ Assel Telesheva \\ Oralbek Karabashev \\ Akhmetzhan Tashkenbayev
}

Kazakh National Technical University, Institute of Industrial Engineering, Almaty, Republic of Kazakhstan

\section{Doi:10.5901/mjss.2014.v5n23p2627}

\section{Abstract}

A solution to the problem of synthesizing an initial three-dimensional kinematic chain with spherical and rotary kinematic pairs is presented. It is shown that this chain can be used as a structural module for structural-kinematic synthesis of threedimensional four-link motion generating lever mechanisms by the preset positions of the in-and output links.

Keywords: mechanism, four-link, kinematic pairs, kinematic chains, synthesis

\section{Introduction}

These papers demonstrate that four-link basic kinematic chains (BKC) may be used as a structural module with structural and kinematic synthesis of plain linkage mechanisms. Such an approach to the synthesis of plain mechanisms allows reducing the problem of their structural and kinematic synthesis to the solution of problem of BKC synthesis, which is very useful for automation of mechanisms engineering. This paper testifies that specified approach may be applied to the problem of structural and kinematic synthesis of spatial linkage mechanisms. The solution of the problem of synthesis of spatial BKC of RSS type (R- rotational, S - spherical kinematic pairs) is represented and its use as a structural module with structural and kinematic synthesis of spatial linkage mechanisms as per predetermined positions of input and output links is shown. A method of solution the problem of BKC synthesis of RSS type is based on the introduction of two movable bodies invariably associated with the input and output links.

\section{Theory}

If two adjacent elements of open four-link BKC with spherical kinematic pairs are tending to infinity, then it is necessary to replace the spherical kinematic pair for the plain or cylindrical.

Proof: If in formula (6) $D_{1}=0$, then $\left(X_{A}, Y_{A}, Z_{A}\right) \rightarrow \infty$ and center of circumference approaching sphere will be laid in the plane or along a straight line. Then, in addition to the required parameters of BKC $B\left(x_{B}, y_{B}, z_{B}\right) \in Q_{1}$ and $C\left(x_{C}, y_{C}, z_{C}\right) \in Q_{2}$ with common parameter $R$, on fixed system of coordinates $O X Y Z$ instead of point $A\left(X_{A}, Y_{A}, Z_{A}\right) \in Q$ it is necessary to determine coefficients $a, b, c$, of plane $Q$.

This section considers a problem of synthesis of spatial basic kinematic chains (BKC) with rotating, plane and spherical kinematic pairs as per specified positions of input and output links based on introduction of two movable solids all the time connected with input and output links[ ${ }^{[1]}$.

Problem statement: Given $N$ of finite distant positions of two solids $Q_{1} \operatorname{n}_{2}: Q_{1}\left(x_{A}, y_{A}, z_{A}, \psi_{1 i}, \theta_{1 i}, \varphi_{1 i}\right)$, 
$Q_{2}\left(x_{D i}, y_{D i}, z_{D i}, \psi_{2 i}, \theta_{2 i}, \varphi_{2 i}\right),(i=\overline{1, N})$,

where, $\psi_{j i}, \theta_{j i}, \varphi_{j i}$ - fixed axis Eulerian angles OXYZ (Figure 1).

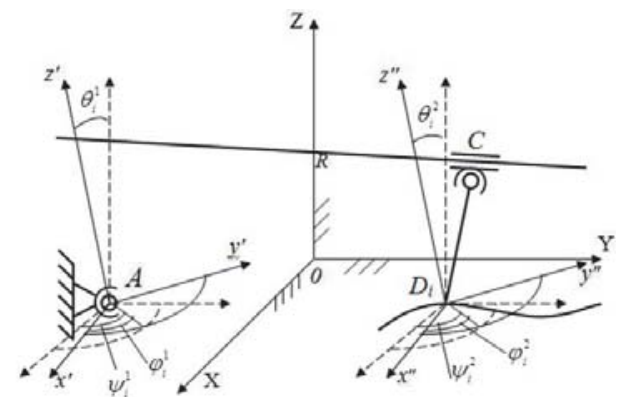

Fig .1 Equivalent four-link kinematic chain ACD.

It is required to find a point $A\left(x_{A}, y_{A}, z_{A}\right)$ on solid $Q$, to find plane on solid $Q_{1}$

$a x+b y+c z+d=0$

and point $C\left(x_{C}, y_{C}, z_{C}\right)$ on solid $Q_{1}$, which in its motion about solid $Q_{1}$ approached to desired plane (1). Equation of plane (1) on fixed solid $Q$ is determined by known transformation formulas

$a\left[\left(X_{C i}-X_{A}\right) t_{11}+\left(Y_{C i}-Y_{A}\right) t_{21}+\left(Z_{C i}-Z_{A}\right) t_{31}\right]+$

$+b\left[\left(X_{C i}-X_{A}\right) t_{12}+\left(Y_{C i}-Y_{A}\right) t_{22}+\left(Z_{C i}-Z_{A}\right) t_{32}\right]+$

$+c\left[\left(X_{C i}-X_{A}\right) t_{13}+\left(Y_{C i}-Y_{A}\right) t_{23}+\left(Z_{C i}-Z_{A}\right) t_{33}\right]+d=0$

where, $\psi_{j i}, \varphi_{j i}$ - angles are given, and angle $\theta_{j i}=0, j=1.2, i=\overline{1, N}$.

$\alpha_{j i}=\psi_{1 i}+\varphi_{1 i}, \beta_{j i}=\psi_{2 i}+\varphi_{2 i}$

$\left[\begin{array}{c}X_{C i} \\ Y_{C i} \\ Z_{C i} \\ 1\end{array}\right]=\left[\begin{array}{cccc}\cos \left(\psi_{2 i}+\varphi_{2 i}\right) & -\sin \left(\psi_{2 i}+\varphi_{2 i}\right) & 0 & X_{D i} \\ \sin \left(\psi_{2 i}+\varphi_{2 i}\right) & \cos \left(\psi_{2 i}+\varphi_{2 i}\right) & 0 & Y_{D i} \\ 0 & 0 & 1 & Z_{D i} \\ 0 & 0 & 0 & 1\end{array}\right] \cdot\left[\begin{array}{c}x_{C} \\ y_{C} \\ z_{C} \\ 1\end{array}\right]$

$t_{11}=\cos \left(\psi_{1 i}+\varphi_{1 i}\right), t_{21}=\sin \left(\psi_{1 i}+\varphi_{1 i}\right), t_{12}=-\sin \left(\psi_{1 i}+\varphi_{1 i}\right)$,

$t_{22}=\cos \left(\psi_{1 i}+\varphi_{1 i}\right), t_{33}=1, t_{13}=t_{31}=t_{23}=t_{32}=t_{41}=t_{42}=t_{43}=0$.

After substitution of the expression (3) in formula (2) and required transformations let us comprise the weighted difference $\Delta q_{i}$ of point $C_{i}\left(x_{C}, y_{C}, z_{C}\right)$ from plane (2) as:

$\Delta q_{i}=G_{1} \cos \alpha_{j i}+G_{2} \sin \alpha_{j i}+G_{3} \cos \left(\alpha_{j i}-\beta_{j i}\right)+G_{4} \sin \left(\alpha_{j i}-\beta_{j i}\right)+G_{5} X_{i}+$

$+G_{6} Y_{i}+G_{7} Z_{i}+G_{8}+G_{9}-1$

where,

$G_{1}=-\left(a X_{A}+b Y_{A}\right), G_{2}=b X_{A}-a Y_{A}, G_{3}=a x_{C}+b y_{C}, G_{4}=a y_{C}-b x_{C}$,

$G_{5}=a, G_{6}=b, G_{7}=c, G_{8}=c z_{C}, G_{9}=-c Z_{A}$.

$\left[\begin{array}{c}X_{i} \\ Y_{i} \\ Z_{i}\end{array}\right]=\left[\begin{array}{ccc}\cos \alpha_{j i} & \sin \alpha_{j i} & 0 \\ -\sin \alpha_{j i} & \cos \alpha_{j i} & 0 \\ 0 & 0 & 1\end{array}\right] \cdot\left[\begin{array}{c}X_{D i} \\ Y_{D i} \\ Z_{D i}\end{array}\right]$

It should be noted that ten required parameters enter into the expression (2), but after normalization of straight-line equation $d=-1$, the nine required parameters remain. These are coefficients of equation of plane $a, b, c$, coordinates $X_{A}, Y_{A}, Z_{A}$, points $A \in Q$ and coordinates ${ }^{x_{C}}, y_{C},{ }^{z_{C}}$, points $C \in Q_{2}$.

Let us comprise sum of squares of weighted difference for $N$ positions

$S=\sum_{i=1}^{N}\left[\Delta q_{i}\right]^{2} \quad(i=\overline{1, N})$ 
Three systems of equations are obtained, the solution of which is written in the following form of relative required parameters ${ }^{[2]}$ :

$\left(X_{A}, Y_{A}, Z_{A}\right)=\frac{1}{D_{1}}\left(D_{X A}, D_{Y A}, D_{Z A}\right)$

Stationary conditions per variables

$\frac{\partial S}{\partial j}=0 \quad\left(j=G_{1}, G_{2}, G_{3}, G_{4}, G_{5}, G_{6}, G_{7}, G_{8}, G_{9}\right) ;$

would result in the following simultaneous linear algebraic equations as $G_{1} \div G_{9}$;

$A \cdot \bar{G}=\bar{B}$

where, matrix elements $A(9,9)$ :

$a_{11}=\sum \cos ^{2} \alpha_{j i}, \quad a_{12}=a_{21}=\frac{1}{2} \sum \sin \alpha_{j i} \cos \alpha_{j i}, a_{13}=a_{31}=\sum \cos \alpha_{j i} \cdot \cos \left(\alpha_{j i}-\beta_{j i}\right)$,

$a_{14}=a_{41}=\sum \cos \alpha_{j i} \cdot \sin \left(\alpha_{j i}-\beta_{j i}\right), a_{15}=a_{51}=\sum X_{i} \cdot \cos \alpha_{j i}, a_{16}=a_{61}=\sum Y_{i} \cdot \cos \alpha_{j i}$,

$a_{17}=a_{71}=\sum Z_{i} \cdot \cos \alpha_{j i}, a_{18}=a_{81}=\sum \cos \alpha_{j i}, a_{19}=a_{91}=\sum \cos \alpha_{j i}, a_{22}=\sum \sin ^{2} \alpha_{j i}$,

$a_{23}=a_{32}=\sum \sin \alpha_{j i} \cdot \cos \left(\alpha_{j i}-\beta_{j i}\right), a_{24}=a_{42}=\sum \sin \alpha_{j i} \cdot \sin \left(\alpha_{j i}-\beta_{j i}\right)$,

$a_{25}=a_{52}=\sum X_{i} \cdot \sin \alpha_{j i}, a_{26}=a_{62}=\sum Y_{i} \cdot \sin \alpha_{j i}, a_{27}=a_{72}=\sum Z_{i} \cdot \sin \alpha_{j i}$,

$a_{28}=a_{82}=\sum \sin \alpha_{j i}, a_{29}=a_{92}=\sum \sin \alpha_{j i}, a_{33}=\sum \cos ^{2}\left(\alpha_{j i}-\beta_{j i}\right)$,

$a_{34}=a_{43}=\frac{1}{2} \sum \sin \left(\alpha_{j i}-\beta_{j i}\right) \cdot \cos \left(\alpha_{j i}-\beta_{j i}\right), a_{35}=a_{53}=\sum X_{i} \cdot \cos \left(\alpha_{j i}-\beta_{j i}\right)$,

$a_{36}=a_{63}=\sum Y_{i} \cdot \cos \left(\alpha_{j i}-\beta_{j i}\right), a_{37}=a_{73}=\sum Z_{i} \cdot \cos \left(\alpha_{j i}-\beta_{j i}\right)$,

$a_{38}=a_{83}=\sum \cos \left(\alpha_{j i}-\beta_{j i}\right) a_{39}=a_{93}=\sum \cos \left(\alpha_{j i}-\beta_{j i}\right), a_{44}=\sum \sin ^{2}\left(\alpha_{j i}-\beta_{j i}\right)$,

$a_{45}=a_{54}=\sum X_{i} \cdot \sin \left(\alpha_{j i}-\beta_{j i}\right) \quad a_{46}=a_{64}=\sum Y_{i} \cdot \sin \left(\alpha_{j i}-\beta_{j i}\right)$

$a_{47}=a_{74}=\sum Z_{i} \cdot \sin \left(\alpha_{j i}-\beta_{j i}\right)^{\prime} a_{48}=a_{84}=a_{49}=a_{94}=\sum \sin \left(\alpha_{j i}-\beta_{j i}\right)$

$a_{55}=\sum X_{i}^{2}, a_{66}=\sum Y_{i}^{2}, a_{77}=\sum Z_{i}^{2}, a_{88}=a_{99}=N, a_{56}=a_{65}=\sum X_{i}^{2}$,

$a_{57}=a_{75}=\sum X_{i} Z_{i}, a_{58}=a_{85}=a_{59}=a_{95}=\sum X_{i}, a_{67}=a_{76}=\sum Y_{i} Z_{i}$,

$a_{68}=a_{86}=a_{69}=a_{96}=\sum Y_{i}, a_{78}=a_{79}=a_{87}=a_{97}=\sum Z_{i}, a_{89}=a_{98}=1$,

$\bar{G}=\left[G_{1}, \ldots, G_{9}\right]^{T}$

$\bar{B}=\left[\sum \cos \alpha_{j i}, \sum \sin \alpha_{j i}, \sum \cos \left(\alpha_{j i}-\beta_{j i}\right), \sum \sin \left(\alpha_{j i}-\beta_{j i}\right), \sum X_{i}, \sum Y_{i}, \sum Z_{i}, N, N\right]^{T}$

Solution of system (5) enables you to determine the required parameters of synthesis. When coordinates of stand $D\left(X_{D}, Y_{D}, Z_{D}\right)$ of output link of slotted link mechanism have fixed values, you can determine nine required parameters of synthesis:

$$
\begin{aligned}
& X_{A}=-\frac{a G_{1}-b G_{2}}{a^{2}+b^{2}}, \quad Y_{A}=-\frac{b G_{1}+a G_{2}}{a^{2}+b^{2}}, \quad Z_{A}=-\frac{G_{9}}{G_{7}}, x_{C}=\frac{a G_{3}-b G_{4}}{a^{2}+b^{2}}, \\
& y_{C}=\frac{a G_{4}+b G_{3}}{a^{2}+b^{2}}, \quad z_{C}=\frac{G_{8}}{G_{7}}, a=G_{5}, b=G_{6}, c=G_{7}, a^{2}+b^{2} \neq 0, c \neq 0 .
\end{aligned}
$$

Therefore, as per assigned positions of input and output links of transfer mechanisms, you can synthesize the spatial slotted link mechanisms of type $R P_{L} S$ ( $R$ - rotational, $P_{L}$ - plane, $S$ - spherical kinematic pairs).

Now, let us consider a matter of choice of normalization of coefficients of equation of plane $a, b, c$. With normalization of $d=-1$, we obtain the weighted difference (3). When entering $a=-1, b=-1, c=-1$ into expression (3), we obtain exact expressions of displacements $\left(\Delta_{i}\right)_{x},\left(\Delta_{i}\right)_{y},\left(\Delta_{i}\right)_{z}$ of points $C_{i}$ along axes $O X, O Y, O Z$, respectively, which are weighted relative to displacement $\Delta_{i}$ along normal line. Therefore, you may not say beforehand which normalization is the best and so it would be reasonable to consider all four events. 
1. Let us assume that $a=-1$. Then weighted difference considering (4) is as follows $\Delta q_{i}=G_{1} \cos \alpha_{j i}+G_{2} \sin \alpha_{j i}+G_{3} \cos \left(\alpha_{j i}-\beta_{j i}\right)+G_{4} \sin \left(\alpha_{j i}-\beta_{j i}\right)+$

$+G_{5} Y_{i}+G_{6} Z_{i}+G_{7}+G_{8}+G_{9}-X_{i}$

where,

$G_{1}=X_{A}-b Y_{A}, G_{2}=Y_{A}+b X_{A}, G_{3}=-x_{C}+b y_{C}, G_{4}=-\left(b x_{C}+y_{C}\right)$,

$G_{5}=b, G_{6}=c, G_{7}=c z_{C}, G_{8}=c Z_{A}, G_{9}=d$.

Based on stationary state condition $S=\sum\left[\Delta q_{i}\right]^{2}$

$\frac{d S}{d j}=0, \quad\left(j=G_{1} \div G_{9}\right)$

considering (6) we obtain the following linear system:

$$
A \cdot \bar{G}=\bar{B}
$$

where, matrix elements $A(9,9)$ :

$$
\begin{aligned}
& a_{11}=\sum \cos ^{2} \alpha_{j i}, \quad a_{12}=a_{21}=\frac{1}{2} \sum \sin \alpha_{j i} \cos \alpha_{j i}, a_{13}=a_{31}=\sum \cos \alpha_{j i} \cdot \cos \left(\alpha_{j i}-\beta_{j i}\right) \text {, } \\
& a_{14}=a_{41}=\sum \cos \alpha_{j i} \cdot \sin \left(\alpha_{j i}-\beta_{j i}\right), a_{15}=a_{51}=\sum Y_{i} \cdot \cos \alpha_{j i}, a_{16}=a_{61}=\sum Z_{i} \cdot \cos \alpha_{j i} \text {, } \\
& a_{17}=a_{71}=\sum \cos \alpha_{j i}, a_{18}=a_{81}=\sum \cos \alpha_{j i}, a_{19}=a_{91}=\sum \cos \alpha_{j i}, a_{22}=\sum \sin ^{2} \alpha_{j i} \text {, } \\
& a_{23}=a_{32}=\sum \sin \alpha_{j i} \cdot \cos \left(\alpha_{j i}-\beta_{j i}\right), a_{24}=a_{42}=\sum \sin \alpha_{j i} \cdot \sin \left(\alpha_{j i}-\beta_{j i}\right) \text {, } \\
& a_{25}=a_{52}=\sum Y_{i} \cdot \sin \alpha_{j i}, a_{26}=a_{62}=\sum Z_{i} \cdot \sin \alpha_{j i}, a_{27}=a_{72}=\sum \sin \alpha_{j i}, \\
& a_{28}=a_{82}=\sum \sin \alpha_{j i}, a_{29}=a_{92}=\sum \sin \alpha_{j i}, a_{33}=\sum \cos ^{2}\left(\alpha_{j i}-\beta_{j i}\right) \text {, } \\
& a_{34}=a_{43}=\frac{1}{2} \sum \sin \left(\alpha_{j i}-\beta_{j i}\right) \cdot \cos \left(\alpha_{j i}-\beta_{j i}\right), a_{35}=a_{53}=\sum Y_{i} \cdot \sin \left(\alpha_{j i}-\beta_{j i}\right) \\
& a_{36}=a_{63}=\sum Z_{i} \cdot \cos \left(\alpha_{j i}-\beta_{j i}\right), a_{37}=a_{73}=\sum \cos \alpha_{j i}\left(\alpha_{j i}-\beta_{j i}\right), a_{38}=a_{83}=\sum \cos \left(\alpha_{j i}-\beta_{j i}\right) a_{39}=a_{93}=\sum \cos \left(\alpha_{j i}-\beta_{j i}\right) \text {, } \\
& a_{44}=\sum \sin ^{2}\left(\alpha_{j i}-\beta_{j i}\right) \text {, } \\
& a_{45}=a_{54}=\sum Y_{i} \cdot \sin \left(\alpha_{j i}-\beta_{j i}\right), a_{46}=a_{64}=\sum Z_{i} \cdot \sin \left(\alpha_{j i}-\beta_{j i}\right) \\
& a_{47}=a_{74}=\sum \sin \left(\alpha_{j i}-\beta_{j i}\right), a_{48}=a_{84}=\sum \sin \left(\alpha_{j i}-\beta_{j i}\right), a_{49}=a_{94}=\sum \sin \left(\alpha_{j i}-\beta_{j i}\right), \\
& a_{55}=\sum Y_{i}^{2}, a_{56}=a_{65}=\sum Y_{i} Z_{i}, a_{57}=a_{75}=\sum Y_{i}, a_{58}=a_{85}=\sum Y_{i}, a_{59}=a_{95}=\sum Y_{i} \text {, } \\
& a_{66}=\sum Z_{i}^{2}, a_{67}=a_{76}=\sum Z_{i}, a_{68}=a_{86}=\sum Z_{i}, a_{69}=a_{96}=\sum Z_{i} \text {, } \\
& a_{77}=a_{88}=a_{99}=N, a_{78}=a_{87}=a_{79}=a_{97}=a_{89}=a_{98}=1 \text {, } \\
& X=\left[G_{1}, G_{2}, G_{3}, G_{4}, G_{5}, G_{6}, G_{7}, G_{8}, G_{9}\right]^{T} \text {, } \\
& \bar{B}=\left[\sum X_{i} \cos \alpha_{j i}, \sum X_{i} \sin \alpha_{j i}, \sum X_{i} \cos \left(\alpha_{j i}-\beta_{j i}\right), \sum X_{i} \sin \left(\alpha_{j i}-\beta_{j i}\right)\right. \text {, } \\
& \left.\sum X_{i} Y_{i}, \sum X_{i} Z_{i}, \sum X_{i}, \sum Y_{i}, \sum Z_{i}\right]^{T} \\
& \text { Solution of system (8) enables you to determine the required parameters of synthesis as: }
\end{aligned}
$$$$
X_{A}=\frac{G_{1}+b G_{2}}{1+b^{2}}, Y_{A}=\frac{G_{2}-b G_{1}}{1+b^{2}}, Z_{A}=-\frac{G_{8}}{G_{6}}, x_{C}=-\frac{G_{3}-b G_{4}}{1+b^{2}},
$$$$
y_{C}=\frac{-G_{4}+b G_{3}}{1+b^{2}}, z_{C}=\frac{G_{7}}{G_{6}}, b=G_{5} \quad, c=G_{6}, d=G_{9} .
$$

As a consequence of solution of this problem, we determine such coordinates of points $A\left(X_{A}, Y_{A}, Z_{A}\right) \in Q$, $C\left(x_{C}, y_{C}, z_{C}\right) \in Q_{2}$, and plane on solid $Q_{1}$ that by combining plane and spherical kinematic pairs with them, we obtain open-link spatial kinematic chain $A C D$ of type $R P_{L} S$ ( $R$ - rotational, $P_{L}$ - plane, $S$ - spherical kinematic pairs).

2. Let us assume that $b=-1$. Then weighted difference considering (4) is as follows 
$\Delta q_{i}=G_{1} \cos \alpha_{j i}+G_{2} \sin \alpha_{j i}+G_{3} \cos \left(\alpha_{j i}-\beta_{j i}\right)+G_{4} \sin \left(\alpha_{j i}-\beta_{j i}\right)+$

$+G_{5} X_{i}+G_{6} Z_{i}+G_{7}+G_{8}+G_{9}-Y_{i}$,

where,

$G_{1}=Y_{A}-a X_{A}, G_{2}=-X_{A}-a Y_{A}, G_{3}=a x_{C}-y_{C}, G_{4}=x_{C}+a y_{C}, G_{5}=a$

$G_{6}=c, G_{7}=c z_{C}, G_{8}=c Z_{A}, G_{9}=d$

$X_{i}=X_{D i} \cos \alpha_{j i}+Y_{D i} \sin \alpha_{j i}, Y_{i}=-X_{D i} \sin \alpha_{j i}+Y_{D i} \cos \alpha_{j i}$

Based on stationary state condition $S=\sum\left[\Delta q_{i}\right]^{2}$

$\frac{d S}{d j}=0 \quad,\left(j=G_{1} \div G_{9}\right)$

considering $\Delta_{q_{i}}$ we obtain the following linear system:

$A \cdot \bar{G}=\bar{B}$

where, matrix elements $A(9,9)$ :

$a_{11}=\sum \cos ^{2} \alpha_{j i}, a_{12}=a_{21}=\frac{1}{2} \sum \sin \alpha_{j i} \cos \alpha_{j i}, a_{13}=a_{31}=\sum \cos \alpha_{j i} \cdot \cos \left(\alpha_{j i}-\beta_{j i}\right)$,

$a_{14}=a_{41}=\sum \cos \alpha_{j i} \cdot \sin \left(\alpha_{j i}-\beta_{j i}\right), a_{15}=a_{51}=\sum X_{i} \cdot \cos \alpha_{j i}, a_{16}=a_{61}=\sum Z_{i} \cdot \cos \alpha_{j i}$,

$a_{17}=a_{71}=\sum \cos \alpha_{j i}, a_{18}=a_{81}=\sum \cos \alpha_{j i}, a_{19}=a_{91}=\sum \cos \alpha_{j i}, a_{22}=\sum \sin ^{2} \alpha_{j i}$,

$a_{23}=a_{32}=\sum \sin \alpha_{j i} \cdot \cos \left(\alpha_{j i}-\beta_{j i}\right) \quad a_{24}=a_{42}=\sum \sin \alpha_{j i} \cdot \sin \left(\alpha_{j i}-\beta_{j i}\right)$,

$a_{25}=a_{52}=\sum X_{i} \cdot \sin \alpha_{j i}, a_{26}=a_{62}=\sum Z_{i} \cdot \sin \alpha_{j i}, a_{27}=a_{72}=\sum \sin \alpha_{j i}$,

$a_{28}=a_{82}=\sum \sin \alpha_{j i}, a_{29}=a_{92}=\sum \sin \alpha_{j i}, a_{33}=\sum \cos ^{2}\left(\alpha_{j i}-\beta_{j i}\right)$,

$a_{34}=a_{43}=\frac{1}{2} \sum \sin \left(\alpha_{j i}-\beta_{j i}\right) \cdot \cos \left(\alpha_{j i}-\beta_{j i}\right), a_{35}=a_{53}=\sum X_{i} \cdot \sin \left(\alpha_{j i}-\beta_{j i}\right)$

$a_{36}=a_{63}=\sum Z_{i} \cdot \cos \left(\alpha_{j i}-\beta_{j i}\right) \quad, \quad a_{37}=a_{73}=\sum \cos \alpha_{j i}\left(\alpha_{j i}-\beta_{j i}\right) \quad, \quad a_{38}=a_{83}=\sum \cos \left(\alpha_{j i}-\beta_{j i}\right)$

$a_{39}=a_{93}=\sum \cos \left(\alpha_{j i}-\beta_{j i}\right) a_{44}=\sum \sin ^{2}\left(\alpha_{j i}-\beta_{j i}\right)$

$a_{45}=a_{54}=\sum X_{i} \cdot \sin \left(\alpha_{j i}-\beta_{j i}\right) \quad a_{46}=a_{64}=\sum Z_{i} \cdot \sin \left(\alpha_{j i}-\beta_{j i}\right)$

$a_{47}=a_{74}=\sum \sin \left(\alpha_{j i}-\beta_{j i}\right)^{\prime}, a_{48}=a_{84}=\sum \sin \left(\alpha_{j i}-\beta_{j i}\right), a_{49}=a_{94}=\sum \sin \left(\alpha_{j i}-\beta_{j i}\right)$,

$a_{55}=\sum X_{i}^{2}, a_{56}=a_{65}=\sum X_{i} Z_{i}, a_{57}=a_{75}=\sum X_{i}, a_{58}=a_{85}=\sum X_{i}, a_{59}=a_{95}=\sum X_{i}$,

$a_{66}=\sum Z_{i}^{2}, a_{67}=a_{76}=\sum Z_{i}, a_{68}=a_{86}=\sum Z_{i}, a_{69}=a_{96}=\sum Z_{i}$,

$a_{77}=a_{88}=a_{99}=N, a_{78}=a_{87}=a_{79}=a_{97}=a_{89}=a_{98}=1$,

$X=\left[G_{1}, G_{2}, G_{3}, G_{4}, G_{5}, G_{6}, G_{7}, G_{8}, G_{9}\right]^{T}$

$\bar{B}=\left[\sum Y_{i} \cos \alpha_{j i}, \sum Y_{i} \sin \alpha_{j i}, \sum Y_{i} \cos \left(\alpha_{j i}-\beta_{j i}\right), \sum Y_{i} \sin \left(\alpha_{j i}-\beta_{j i}\right)\right.$,

$\left.\sum Y_{i} Y_{i}, \sum Y_{i} Z_{i}, \sum Y_{i}, \sum Y_{i}, \sum Y_{i}\right]^{T}$

System solution enables to determine the required parameters as:

$X_{A}=\frac{-G_{2}-a G_{1}}{1+a^{2}}, Y_{A}=\frac{-a G_{2}+G_{1}}{1+a^{2}}, Z_{A}=-\frac{G_{8}}{G_{6}}, x_{C}=\frac{a G_{3}+G_{4}}{1+a^{2}}, y_{C}=\frac{a G_{4}-G_{3}}{1+a^{2}}, z_{C}=\frac{G_{7}}{G_{6}}, b=G_{5}, c=G_{6}, d=G_{9}$.

As a consequence of solution of this problem, we determine such coordinates of points $A\left(X_{A}, Y_{A}, Z_{A}\right) \in Q$, $C\left(x_{C}, y_{C}, z_{C}\right) \in Q_{2}$, and plane on solid $Q_{1}$ that by combining plane and spherical kinematic pairs with them, we obtain open-link spatial kinematic chain $A C D$ of type $R P_{L} S$ ( $R$ - rotational, $P_{L}$ - plane, $S$ - spherical kinematic pairs).

3. Let us assume that $c=-1$. Then weighted difference considering (4) is as follows

$\Delta q_{i}=G_{1} \cos \alpha_{j i}+G_{2} \sin \alpha_{j i}+G_{3} \cos \left(\alpha_{j i}-\beta_{j i}\right)+G_{4} \sin \left(\alpha_{j i}-\beta_{j i}\right)+$ 
$+G_{5} X_{i}+G_{6} Y_{i}+G_{7}+G_{8}+G_{9}-Z_{D i}$

where,

$G_{i}=-a X_{A}-6 Y_{A}, G_{2}=b X_{A}-a Y_{A}, G_{3}=a x_{C}+b y_{C}, G_{4}=\left(a y_{C}-b x_{C}\right), G_{5}=a$, $G_{6}=b, G_{7}=z_{C}, G_{8}=Z_{A}, G_{9}=d$.

Based on stationary state condition $S=\sum\left[\Delta q_{i}\right]^{2}$

$\frac{d S}{d j}=0,\left(j=G_{1} \div G_{9}\right)$

considering $\Delta_{q_{i}}$ we obtain the following linear system:

$A \cdot \bar{G}=\bar{B}$

where, matrix elements $A(9,9)$ :

$a_{11}=\sum \cos ^{2} \alpha_{j i}, a_{12}=a_{21}=\frac{1}{2} \sum \sin \alpha_{j i} \cos \alpha_{j i}, a_{13}=a_{31}=\sum \cos \alpha_{j i} \cdot \cos \left(\alpha_{j i}-\beta_{j i}\right)$

$a_{14}=a_{41}=\sum \cos \alpha_{j i} \cdot \sin \left(\alpha_{j i}-\beta_{j i}\right), a_{15}=a_{51}=\sum X_{i} \cdot \cos \alpha_{j i}, a_{16}=a_{61}=\sum Y_{i} \cdot \cos \alpha_{j i}$,

$a_{17}=a_{71}=\sum \cos \alpha_{j i}, a_{18}=a_{81}=\sum \cos \alpha_{j i}, a_{19}=a_{91}=\sum \cos \alpha_{j i}, a_{22}=\sum \sin ^{2} \alpha_{j i}$,

$a_{23}=a_{32}=\sum \sin \alpha_{j i} \cdot \cos \left(\alpha_{j i}-\beta_{j i}\right), a_{24}=a_{42}=\sum \sin \alpha_{j i} \cdot \sin \left(\alpha_{j i}-\beta_{j i}\right)$,

$a_{25}=a_{52}=\sum X_{i} \cdot \sin \alpha_{j i}, a_{26}=a_{62}=\sum Y_{i} \cdot \sin \alpha_{j i}, a_{27}=a_{72}=\sum \sin \alpha_{j i}$,

$a_{28}=a_{82}=\sum \sin \alpha_{j i}, a_{29}=a_{92}=\sum \sin \alpha_{j i}, a_{33}=\sum \cos ^{2}\left(\alpha_{j i}-\beta_{j i}\right)$,

$a_{34}=a_{43}=\frac{1}{2} \sum \sin \left(\alpha_{j i}-\beta_{j i}\right) \cdot \cos \left(\alpha_{j i}-\beta_{j i}\right), a_{35}=a_{53}=\sum X_{i} \cdot \cos \left(\alpha_{j i}-\beta_{j i}\right)$

$a_{36}=a_{63}=\sum Y_{i} \cdot \cos \left(\alpha_{j i}-\beta_{j i}\right) \quad, \quad a_{37}=a_{73}=\sum \cos \alpha_{j i}\left(\alpha_{j i}-\beta_{j i}\right) \quad, \quad a_{38}=a_{83}=\sum \cos \left(\alpha_{j i}-\beta_{j i}\right)$

$a_{39}=a_{93}=\sum \cos \left(\alpha_{j i}-\beta_{j i}\right) a_{44}=\sum \sin ^{2}\left(\alpha_{j i}-\beta_{j i}\right)$,

$a_{45}=a_{54}=\sum X_{i} \cdot \sin \left(\alpha_{j i}-\beta_{j i}\right), a_{46}=a_{64}=\sum Y_{i} \cdot \sin \left(\alpha_{j i}-\beta_{j i}\right)$

$a_{47}=a_{74}=\sum \sin \left(\alpha_{j i}-\beta_{j i}\right), a_{48}=a_{84}=\sum \sin \left(\alpha_{j i}-\beta_{j i}\right), a_{49}=a_{94}=\sum \sin \left(\alpha_{j i}-\beta_{j i}\right)$,

$a_{55}=\sum X_{i}^{2}, a_{56}=a_{65}=\sum X_{i} Y_{i}, a_{57}=a_{75}=\sum X_{i}, a_{58}=a_{85}=\sum X_{i}, a_{59}=a_{95}=\sum X_{i}$,

$a_{66}=\sum Y_{i}^{2}, a_{67}=a_{76}=\sum Y_{i}, a_{68}=a_{86}=\sum Y_{i}, a_{69}=a_{96}=\sum Y_{i}$,

$a_{77}=a_{88}=a_{99}=N, \quad a_{78}=a_{87}=a_{79}=a_{97}=a_{89}=a_{98}=1$,

$X=\left[G_{1}, G_{2}, G_{3}, G_{4}, G_{5}, G_{6}, G_{7}, G_{8}, G_{9}\right]^{T}$

$\bar{B}=\left[\sum Z_{D i} \cos \alpha_{j i}, \sum Z_{D i} \sin \alpha_{j i}, \sum Z_{D i} \cos \left(\alpha_{j i}-\beta_{j i}\right), \sum Z_{D i} \sin \left(\alpha_{j i}-\beta_{j i}\right)\right.$,

$\left.\sum Z_{D i} X_{i}, \sum Z_{D i} Y_{i}, \sum Z_{D i}, \sum Z_{D i}, \sum Z_{D i}\right]^{T}$

System solution enables to determine the required parameters as:

$$
\begin{array}{lll}
X_{A}=\frac{b G_{2}-a G_{1}}{a^{2}+b^{2}}, & Y_{A}=-\frac{b G_{1}+a G_{2}}{a^{2}+b^{2}}, & Z_{A}=G_{8}, \quad x_{C}=\frac{a G_{3}-b G_{4}}{a^{2}+b^{2}}, \\
y_{C}=\frac{a G_{4}+b G_{3}}{a^{2}+b^{2}}, & z_{C}=G_{7} a=G_{5} \quad, b=G_{6}, d=G_{9} .
\end{array}
$$

As a consequence of solution of this problem, we determine such coordinates of points $A\left(X_{A}, Y_{A}, Z_{A}\right) \in Q$, $C\left(x_{C}, y_{C}, z_{C}\right) \in Q_{2}$ and plane on solid $Q_{1}$ that by combining plane and spherical kinematic pairs with them, we obtain open-link spatial kinematic chain $A C D$ of type $R P_{L} S$ ( $R$ - rotational, $P_{L}$ - plane, $S$ - spherical kinematic pairs).

In the cases of normalization $d=-1$ the course of problem solution remains analogical.

By fixing or specifying a part of the required parameters of synthesis in various combinations, we may obtain different modifications of BKC with the plane and spherical pairs: 
a) Let there be given coordinates ${ }^{X_{A i}}, Y_{A i}, Z_{A i}$ of point $A$, coordinates $X_{D i}, Y_{D i}, Z_{D i}$ of point $D$ and Euler angles $Q_{1}\left(\psi_{1 i}, \theta_{1 i}, \varphi_{1 i}\right), Q_{2}\left(\psi_{2 i}, \theta_{2 i}, \varphi_{2 i}\right)$. Then as result of synthesis, we can obtain three-link spatial BKC ACD.

$\Delta q_{i}=G_{1} \cos \left(\alpha_{j i}-\beta_{j i}\right)+G_{2} \sin \left(\alpha_{j i}-\beta_{j i}\right)+G_{3} X_{i}++G_{4} Y_{i}+G_{5} Z_{i}+G_{6}-1$,

where,

$G_{1}=\left(a x_{C}+b y_{C}\right), G_{2}=a y_{C}-b x_{c}, G_{3}=a, G_{4}=8, G_{5}=c, G_{6}=c z_{C}$.
$\left[\begin{array}{c}X_{i} \\ Y_{i} \\ Z_{i}\end{array}\right]=\left[\begin{array}{ccc}\cos \alpha_{j i} & \sin \alpha_{j i} & 0 \\ -\sin \alpha_{j i} & \cos \alpha_{j i} & 0 \\ 0 & 0 & 1\end{array}\right] \cdot\left[\begin{array}{c}X_{D i}-X_{A i} \\ Y_{D i}-Y_{A i} \\ Z_{D i}-Z_{A i}\end{array}\right]$

Let us comprise sum of squares of weighted difference for $N$ positions

$S=\sum_{i=1}^{N}\left[\Delta q_{i}\right]^{2} \quad(i=1, N)$

Stationary conditions per variables

$\frac{\partial S}{\partial j}=0 \quad\left(j=G_{1}, G_{2}, G_{3}, G_{4}, G_{5}, G_{6}\right)$;

would result in the following simultaneous linear algebraic equations as $G_{1} \div G_{6}$;

$A \cdot \bar{G}=\bar{B}$

where, matrix elements $A(6,6)$ :

$a_{11}=\sum \cos ^{2}\left(\alpha_{j i}-\beta_{j i}\right) \quad, \quad a_{12}=a_{21}=\frac{1}{2} \sum \sin \left(\alpha_{j i}-\beta_{j i}\right) \cdot \cos \left(\alpha_{j i}-\beta_{j i}\right) \quad, \quad a_{13}=a_{31}=\sum X_{i} \cos \left(\alpha_{j i}-\beta_{j i}\right)$ $a_{14}=a_{41}=\sum Y_{i} \cos \left(\alpha_{j i}-\beta_{j i}\right)$

$a_{15}=a_{51}=\sum Z_{i} \cos \left(\alpha_{j i}-\beta_{j i}\right), a_{16}=a_{61}=\sum \cos \left(\alpha_{j i}-\beta_{j i}\right)$

$a_{22}=\sum \sin ^{2}\left(\alpha_{j i}-\beta_{j i}\right), a_{23}=a_{32}=\sum X_{i} \sin \left(\alpha_{j i}-\beta_{j i}\right)$,

$a_{24}=a_{42}=\sum Y_{i} \sin \left(\alpha_{j i}-\beta_{j i}\right), a_{25}=a_{52}=\sum Z_{i} \sin \left(\alpha_{j i}-\beta_{j i}\right)$,

$a_{26}=a_{62}=\sum \sin \left(\alpha_{j i}-\beta_{j i}\right), a_{33}=\sum X_{i}^{2}, \quad a_{34}=a_{43}=\sum X_{i} Y_{i} \quad a_{35}=a_{53}=\sum X_{i} Z_{i}, a_{36}=a_{63}=\sum X_{i}$,

$a_{44}=\sum Y_{i}^{2}, a_{45}=a_{54}=\sum Y_{i} Z_{i}, a_{46}=a_{64}=\sum Y_{i}$

$a_{55}=\sum Z_{i}^{2}, a_{56}=a_{65}=\sum Z_{i}, a_{66}=N$,

$X=\left[G_{1}, G_{2}, G_{3}, G_{4}, G_{5}, G_{6}, G_{7}, G_{8}, G_{9}\right]^{T}$.

$\bar{B}=\left[\sum \cos \left(\alpha_{j i}-\beta_{j i}\right), \sum \sin \left(\alpha_{j i}-\beta_{j i}\right), \sum X_{i}, \sum Y_{i}, \sum Z_{i}, N\right]^{T}$

System solution enables you to determine the required parameters of synthesis as:

$x_{C}=\frac{a G_{1}-b G_{2}}{a^{2}+b^{2}}, y_{C}=\frac{b G_{1}+a G_{2}}{a^{2}+b^{2}}, \quad z_{C}=\frac{G_{6}}{G_{5}}, a=G_{3} \quad, b=G_{4} \quad c=G_{5}, a^{2}+b^{2} \neq 0$.

б) If Euler angles $\psi_{1 i}=\theta_{1 i}=\varphi_{1 i}=0$ of solid $Q_{1}$, then a problem reduces to the weighted square approximation of point $C_{i}\left(x_{C}, y_{C}, z_{C}\right)$ with $N$ approximate-collinear positions, etc.

Example: Let us consider problem of synthesis of spatial four-link mechanism of type $R P_{L} S$ ( $R$-rotational, $P_{L}-$ plane, $S$ - spherical kinematic pairs). Approximately reproducing function

$\psi=-50 \cos \frac{6}{5} \varphi, \varphi \in\left[0^{0}, 120^{\circ}\right]$. We divide an interval $\left[0^{\circ}, 120^{\circ}\right]$ into 20 equal parts (Figure 2). If we are given axial angle $\rho=90^{\circ}$ and coordinates $X_{D}=Z_{D}=0, Y_{D}=1,2$ of point $D$, let us determine the following parameters of fourlink chain $A B C D$ :

$$
a=\sqrt{x_{B}^{2}+y_{B}^{2}+z_{B}^{2}}, b=R, c=\sqrt{x_{C}^{2}+y_{C}^{2}+z_{C}^{2}}, X_{A}, Y_{A}, Z_{A}, \varphi_{0}=\arccos \frac{z_{B}}{a}, \psi_{0}=\arccos \frac{z_{C}}{c} .
$$




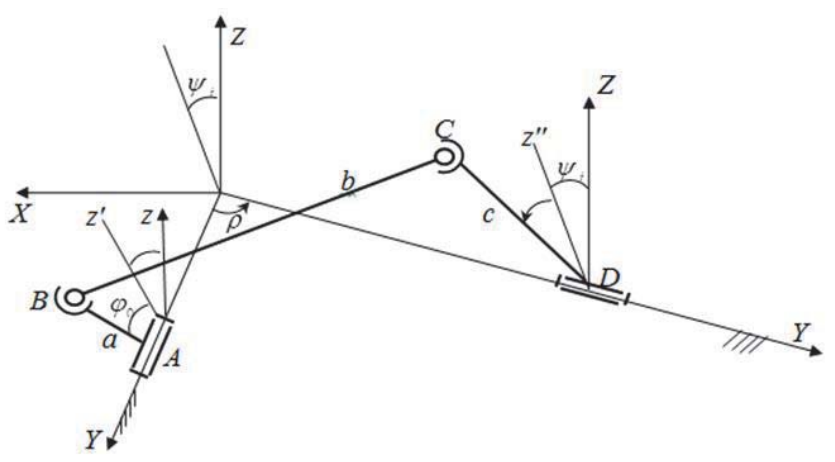

Fig. 2 Four link spatial chain $A B C D$.

For synthesis of this problem, we use expression (2). Then matrix is as follows:

$T_{j k}^{i}=T_{01} \cdot T_{02} \cdot T_{03}$,

where,

$$
T_{01}=\left[\begin{array}{ccc}
\cos \varphi_{i} & 0 & -\sin \varphi_{i} \\
0 & 1 & 0 \\
\sin \varphi_{i} & 0 & \cos \varphi_{i}
\end{array}\right], T_{02}=\left[\begin{array}{ccc}
\cos \rho & -\sin \rho & 0 \\
\sin \rho & \cos \rho & 0 \\
0 & 0 & 1
\end{array}\right], T_{03}=\left[\begin{array}{ccc}
\cos \psi_{i} & 0 & \sin \psi_{i} \\
0 & 1 & 0 \\
-\sin \psi_{i} & 0 & \cos \psi_{i}
\end{array}\right]
$$

On order to determine the required parameters of synthesis, we use search algorithm of minimum of sum $S$. Based on above minimization algorithm, the value of minimum of sum $S=0.00012$.

Then a problem of mechanism synthesis reduces to minimization of the objective function of BKC

$S=\sum_{i=1}^{21}\left[\Delta_{q i}\left(X_{A}, Y_{A}, Z_{A}, x_{B}, y_{B}, z_{B}, R, x_{C}, y_{C}, z_{C}\right)\right]^{2}$

For solution of this problem we apply the above given search algorithm of minimum of sum $S$. According to algorithm, we are given 10 values $B\left(x_{B}^{(0)}, y_{B}^{(0)}, z_{B}^{(0)}\right){ }_{n} C\left(x_{C}^{(0)}, y_{C}^{(0)}, z_{C}^{(0)}\right)$ depending on the length of links $a$ and $c$. For each preliminary value of points $B\left(x_{B}^{(0)}, y_{B}^{(0)}, z_{B}^{(0)}\right)$ and $C\left(x_{C}^{(0)}, y_{C}^{(0)}, z_{C}^{(0)}\right)$ in Table 1 are given the results of calculations.

Iteration process of minimum search of function $S$ is completed upon satisfaction of inequation

$$
\left|R^{(k)}-R^{(k-1)}\right| \leq \mathcal{E},\left|X_{A}^{(k)}-X_{A}^{(k-1)}\right| \leq \mathcal{E},\left|Y_{A}^{(k)}-Y_{A}^{(k-1)}\right| \leq \mathcal{E},\left|Z_{A}^{(k)}-Z_{A}^{(k-1)}\right| \leq \mathcal{E} \text {, where, } \varepsilon=10^{-4} \text {. }
$$

\section{Results}

Table 1: Figures $3-6$ demonstrate $2 D$ and $3 D$ graphics of objective function $S$.

\begin{tabular}{cccccccccccc}
\hline \hline$N$ & $x_{B}$ & $y_{B}$ & $z_{B}$ & $R$ & $x_{C}$ & $y_{C}$ & $z_{C}$ & $X_{A}$ & $Y_{A}$ & $Z_{A}$ & $S$ \\
\hline \hline 1 & 1,014 & 1,815 & 1,2968 & 2,6406 & 0,804 & 1,9819 & 0,3277 & 0,0001 & 1,204 & 0 & 0,00152 \\
\hline 2 & 0,9178 & 1,7169 & 0,8219 & 2,41518 & 0,9017 & 1,7618 & 0,3838 & 0,0002 & 1,105 & 0 & 0,00103 \\
\hline 3 & $-1,1171$ & 1,6449 & 0,7263 & 1,9678 & 0,7664 & 0,9261 & 0,4147 & 0 & 1,086 & 0,0001 & 0,00122 \\
\hline 4 & $-0,3181$ & 1,4716 & 0,9227 & 1,7646 & 0,5464 & 0,8167 & 0,4386 & 0 & 1,2667 & 0,0001 & 0,00052 \\
\hline 5 & $-0,22763$ & 1,21314 & 0,609801 & 1,69127 & 0,652906 & 0,639147 & 0,469694 & 0 & 1,189776 & 0 & 0,00012 \\
\hline 6 & 0,7819 & 1,0449 & 0,3233 & 1,5118 & 0,5917 & 0,6221 & 0,4554 & 0 & 1,0997 & 0,0001 & 0,00018 \\
\hline 7 & 0,1918 & 1,1667 & 0,2218 & 1,7246 & 0,4928 & 0,6046 & 0,7184 & 0,0003 & 1,6756 & 0 & 0,00021 \\
\hline 8 & 0,2128 & 1,0717 & 0,3486 & 1,54714 & 0,5154 & 0,7654 & 0,6656 & 0,0002 & 1,7617 & 0 & 0,00146 \\
\hline 9 & 0,56179 & 1,00818 & 0,9417 & 1,3349 & 0,3426 & 0,6406 & 0,6846 & 0,0001 & 1,3606 & 0,0002 & 0,0019 \\
\hline 10 & 0,86718 & 1,2617 & 0,6461 & 1,2667 & 0,5617 & 0,7627 & 0,6976 & 0,0001 & 1,1415 & 0,000 & 0,00031 \\
\hline \hline
\end{tabular}




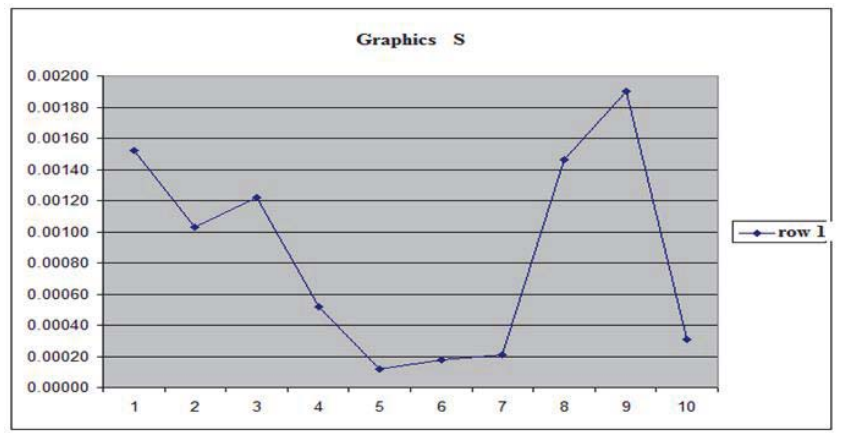

Fig .3 Number of points $S$.

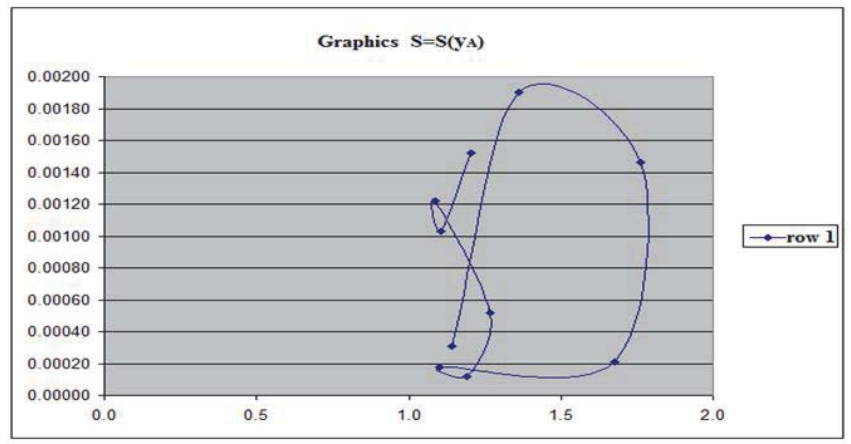

Fig .4 Number of function points $S=S\left(Y_{A}\right)$.

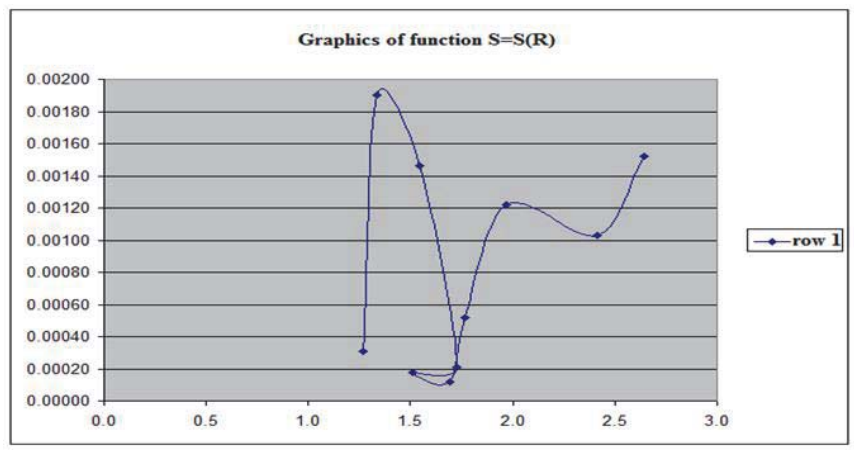

Fig . 5 Number of function points $S=S(R)$. 


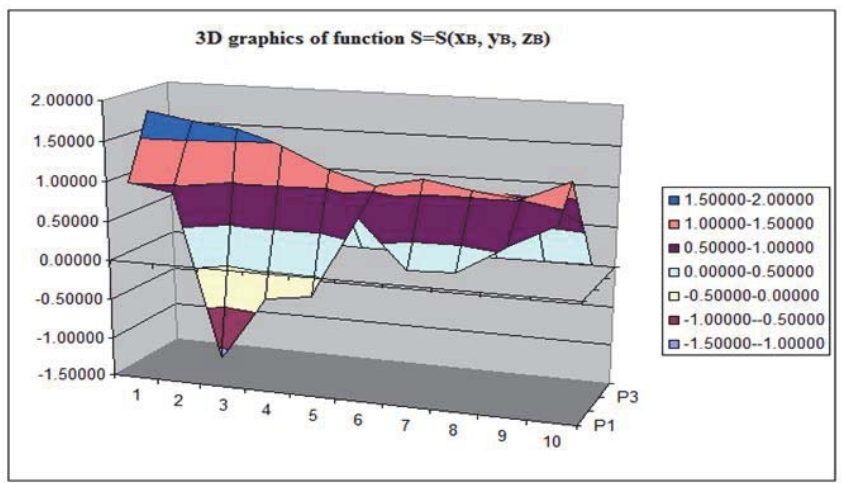

Fig .6 3D graphics of function $S=S\left(x_{B}, y_{B}, z_{B}\right)$.

Values of required parameters of synthesized mechanism:

$x_{B}=-0,227631, x_{C}=0,652906, y_{B}=1,21314, y_{C}=0,639147, z_{B}=0,609801$, $z_{C}=0,469694, X_{A}=Z_{A}=0, Y_{A}=1,189776, R=1,69127$

\section{Discussion}

Thus, the use of one and the same objective function being generated for the synthesis of BKC and its modification, allows automating the process of synthesis of spatial linkage mechanisms as per predetermined positions of input and output links of the mechanism.

\section{Conclusion}

In summary, when there is a synthesis of BKC with spherical kinematic pairs as per predetermined positions of the input and output links of the mechanism, and when two adjacent links of BKC are tending to infinity, it is necessary to replace the spherical kinematic pair for plain or cylindrical. In this case, the synthesized mechanism takes a form of spatial link mechanism after determining the required parameters.

\section{References}

Kosbolov S., Moldabekov M., Bekenov E. Kinematic synthesis of spatial lever motion - generating mechanisms by use of initial kinematic chain. The NINTH IFToMM international symposium on teori of machines and mechanisms. - Bucharest, Romania, September 1-4, 2005. SYROM 2005. -P .245-250.

Kosbolov S., Tanzharikova G., Zhauyt A., Rakhmatulina A. Kinematical synthesis of six-link linkage with three dwells. - Vestnik KazNTU, Almaty, Kazakhstan. - №6(94). 2012. - p. 44-52.

McCarthy J.M. The synthesis of planar RR and spatial CC chains and the equation of a triangle.

Trans. ASME. J. Mech. Des. 1995, Suppl. "50th anniv. des. eng. div." - p. 101-106.

Golynski Z. Optimal synthesis problems solved by means of nonlinear programming and random methods. Journal of mechanisms. Vol. 5. - №3. 1970. - p. 287-309.

Innocenti C. Direct kinematics in analytical form of the 6-4 fully - parallel mechanisms. Trans. ASME. J. Mech. Des. - 1995, 117.- №1.p. 85-95.

Fenton R.G. and Jinshu Xu. Kinematics Synthesis of Mechanisms Using the Flexible Toleranse Algorithm. Proc. of the 7-th World Congress on the Theory of Machines and Mechanisms. -Seville, Spain, 1987. -P. 37-40.

Song S.M., Waldron K.J. Geometric Design of a Walking Machine for Optimal Mobility. Trans of the ASME Journal of Mech., Trans, and Autom. in Design. March. -1987. -V.109. -P.21-28.

Rao S.S. and Ambekar A.G. Optimum design of spherical 4-R function generating mechanisms. Mechanisms and machine theory, Autumn, 1974, vol.9, №3. -P. 405-410.

Culafic G.D., Pantelic T.L. Technological approximative synthesis of a path generating mechanism. Proc. of the 7-th World congress on the theory of machines and mechanisms. -Seville, Spain, 1987. -P. 119-124.

Han Chi-Yeh. A general method for the optimal design of mechanisms. Journal of mechanisms. -1966. -Vol. 1. -P. 301-312. 
Handra-Luka V. The synthesis of mechanisms on the basis of positional shifts determination of the output links. Proc. of the 7-th World congress on the theory of machines and mechanisms. - Seville, Spain, 1987. -P. 99-102.

Klein Breteler A.J. Partial derivatives in kinematic optimization. Proc. of 5-th World Congress on the theory of machines and mechanisms. -Montreal, Canada, 1979.

Tomas J. The synthesis of mechanisms as a nonlinear programming problem journal of mechanisms. -1968. Vol. 3. -P. 119-130 